\title{
Local consonance and the relationship between timbre and scale
}

\author{
William A. Sethares \\ Electrical and Computer Engineering, University of Wisconsin, 1415 Johnson Drive, Madison, \\ Wisconsin 53706
}

(Received 30 November 1992; accepted for publication 25 May 1993)

\begin{abstract}
The principle of local consonance is based on an explicit parametrization of Plomp and Levelt's [J. Acoust. Soc. Am. 38, 548-560 (1965)] consonance curves. It explains the relationship between the spectrum of a sound (its timbre) and a tuning (or scale) in which the timbre will appear most consonant. This relationship is defined in terms of the local minima of a family of dissonance curves. For certain timbres with simple spectral configurations, dissonance curves can be completely characterized, and bounds are provided on the number and location of points of local consonance. Computational techniques are presented which answer two complementary questions: Given a timbre, what scale should it be played in? Given a desired scale, how can appropriate timbres be chosen? Several concrete examples are given, including finding scales for nonharmonic timbres (the natural resonances of a uniform beam, "stretched" and "compressed" timbres, FM timbres with noninteger carrier-to-modulation ratios), and finding timbres for arbitrary scales.
\end{abstract}

PACS numbers: $43.75 . \mathrm{Bc}, 43.66 . \mathrm{Jh}$

\section{INTRODUCTION}

Carlos observed in Ref. 1 that "the timbre of an instrument strongly affects what tuning and scale sound best on that instrument." Interpreting "timbre" to mean spectral decomposition and "sounds best" to refer to the tonal consonance theory of Plomp and Levelt, ${ }^{2}$ this paper establishes a concrete relationship between the timbre of a sound and a family of intervals (a scale) in which the sound will appear most consonant.

A musical interval is generally considered to be consonant if it sounds pleasant or restful; a consonant interval has little or no musical tension or tendency to change. Dissonance is the degree to which an interval sounds unpleasant or rough; dissonant intervals generally feel tense and unresolved. The auditory system typically perceives certain intervals as consonant (such as the octave and fifth) while other intervals are perceived as dissonant (such as the minor second or major seventh). Noting that the consonant intervals tend to be associated with simple ratios of frequency suggests that people might naturally be biased toward such "simple" intervals.

In the previous century, Helmholtz $z^{3}$ suggested that the perception of consonance and dissonance could be understood in terms of the presence or absence of rapid beating between the sinusoidal components of a complex tone. Plomp and Levelt ${ }^{2}$ concretized this in a series of experiments in which subjects reported the relative consonance of intervals of pure sine waves. The resulting curves exhibit none of the complexities expected from studies conducted with musical timbres. Figure 1 shows a family of such curves (which are averaged curves obtained from Ref. 2), each curve covering a different frequency range. All have the same qualitative properties: They begin at unison with high consonance (which appears in Fig. 1 as zero dissonance), and rise rapidly to a maximum dissonance, then slowly decrease. This argues against a natural preference for simple ratios, since the thirds, fourths, fifths, and octaves are undistinguished in these plots.

Plomp and Levelt explained perception of musical intervals by noting that most "musical tones" have a spectrum consisting of a root or fundamental frequency, and a series of partials that occur at integer multiples of the fundamental as in Fig. 2. Assuming that the total dissonance between two complex tones is the sum of the dissonances of all the sinusoidal components leads to the curve in Fig. 3 (see Ref. 4 for an alternate method of combining the sinusoidal dissonance curves). Observe that this curve, which we call the dissonance curve generated by the timbre of Fig. 2, contains major dips at intervals of a fifth and an octave. It also has local minima at most of the 12-tone equal-tempered scale degrees: The major and minor thirds, the fourth, the major sixth, and the seventh (scale steps 4, 3,9 , and 10 , respectively). Thus intervals which occur at points of local minima in the dissonance curve are perceived as relatively consonant.

This correlation between consonant intervals and the points of local minima of the dissonance curve suggests two interesting avenues of investigation. Given an arbitrary timbre $T$ (perhaps one whose spectrum does not consist of a standard harmonic series), it is straightforward to draw the dissonance curve generated by $T$. The local minima of this curve occur at values which are good candidates for notes of a scale, since they are (locally) points of minimum dissonance (or maximum consonance). Alternatively, given a desired scale (perhaps one which divides the octave into $n$ equal pieces, or one which is not based on the octave), one can find timbres which will generate a dissonance curve that contains local minima at precisely these scale degrees.

The relationship between timbre and scale was first explored in Pierce's brief note, ${ }^{5}$ which reported synthesizing a timbre designed specifically to be played in an 8-tone 


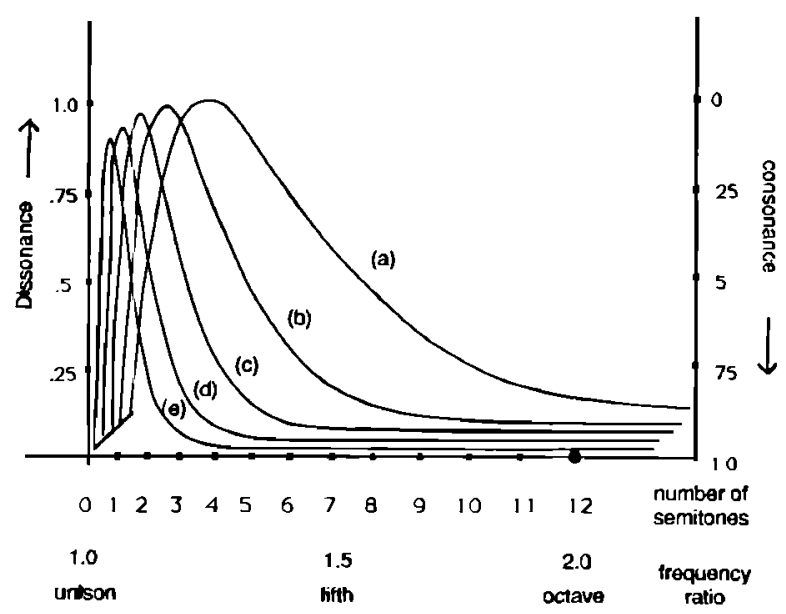

Dissonance curves for pure sine waves as a function of frequency difference. The consonance and dissonance scales are arbitrary

(a) base trequency 125

(b) base Irequency 250

(c) base trequency 500

(d) base frequency 1000

(e) base trequency 2000

FIG. 1. Dissonance curves for pure sine waves as a function of frequency difference. The consonance and dissonance scales are arbitrary: (a) base frequency 125 , (b) base frequency 250 , (c) base frequency 500 , (d) base frequency 1000 , (e) base frequency 2000 .

equal-tempered scale. Pierce concludes, “... by providing music with tones having accurately specified but nonharmonic partials, the digital computer can release music from the tyranny of 12 tones without throwing consonance overboard." Slaymaker ${ }^{6}$ investigated timbres with stretched (and compressed) partials, and Mathews and Pierce ${ }^{7}$ explored their potential musical uses. Geary's study ${ }^{8} \mathrm{em}$ ployed quantitative methods to demonstrate the existence of consonance and dissonance with nonharmonic timbres. Recently, Mathews and Pierce' examined a scale with steps based on $\sqrt[13]{3}$ (rather than the standard $\sqrt[12]{2}$ ) which is designed to be played with timbres containing only odd partials. Carlos ${ }^{1}$ investigated scales for nonharmonic timbres by overlaying their spectra and searching for intervals in which partials coincide, thus minimizing the beats (or roughness) of the sound. This is similar to the present approach, but we provide a systematic technique that can be used to find scales for a given timbre, or to find timbres for a given scale.

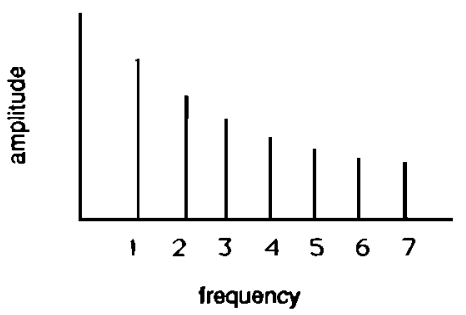

FIG. 2. The standard harmonic timbre used to generate the dissonance curve of Fig. 3. Amplitudes fall at a rate of 0.88 . The frequency axis is normalized so that the root frequency is unity.

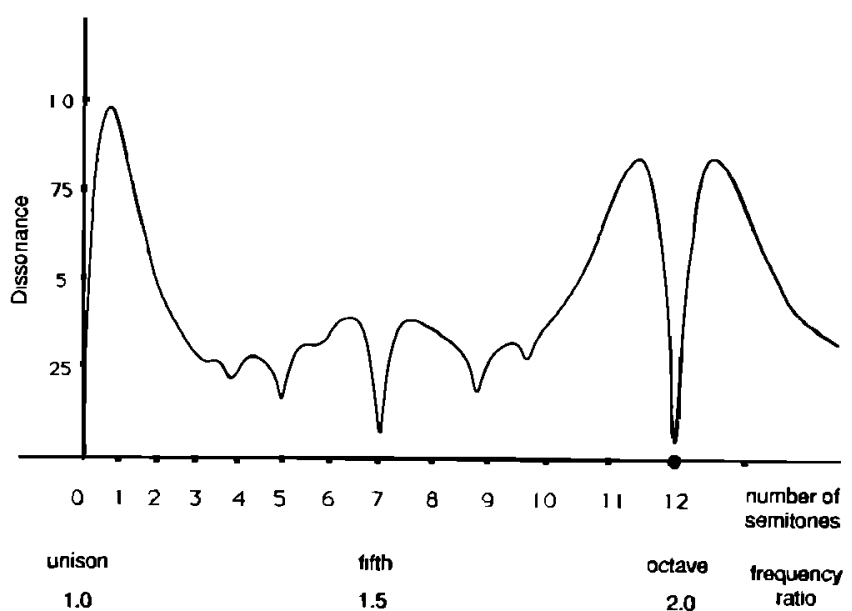

FIG. 3. Dissonance curve for the timbre of Fig. 2. The horizontal axis represents frequency difference. Dots mark the location of notes in the standard 12-tone equal-tempered scale. The vertical axis is arbitrary, and all dissonance curves are normalized so that the largest value occurs at unity.

To make the discussion more concrete, Sec. I parametrizes the Plomp-Levelt curves with a model that is used throughout the paper. This model is studied in detail in the second section, which explores the principle of local consonance and derives results about the existence and placement of local minima. This leads, in Sec. III, to a way of calculating the best scale to use with an arbitrary timbre. Section IV examines the complementary question: How to construct appropriate timbres given an arbitrary scale. The final section summarizes, discusses the implications of the principle of local consonance, and makes suggestions for further experimentation. A simpler (nonmathematical) view of the timbre-scale relationship can be found in Ref. 10.

\section{A QUANTITATIVE MEASURE OF DISSONANCE}

The Plomp-Levelt curves of Fig. 1 can be conveniently parametrized by a model of the form

$$
d(x)=e^{-a x}-e^{-b x},
$$

where $x$ represents the difference in frequency between two sinusoids, and $a$ and $b$ determine the rates at which the function rises and falls. Using a gradient minimization of the squared error between the (averaged) data and the curve $d(x)$ gives values of $a=3.5$ and $b=5.75$.

The dissonance function $d(x)$ can also be scaled so that the curves for different base frequencies and with different amplitudes are represented conveniently. If the point of maximum dissonance occurs at $d^{*}$, then the dissonance between sinusoids at frequency $f_{1}$ with amplitude $v_{1}$ and at frequency $f_{2}$ with amplitude $v_{2}$ (for $f_{1}<f_{2}$ ) is

$$
d\left(f_{1}, f_{2}, v_{1}, v_{2}\right)=v_{12}\left(e^{-a s\left(f_{2}-f_{1}\right)}-e^{-b s\left(f_{2}-f_{1}\right)}\right),
$$

where

$$
s=d^{*} /\left(s_{1} f_{1}+s_{2}\right)
$$

and 


$$
v_{12}=v_{1} v_{2} \text {. }
$$

The form of Eq. (4) ensures that softer components contribute less to the total dissonance measure than those with larger amplitudes. For instance, if either $v_{1}$ or $v_{2}$ approaches zero, then $v_{12}$ decreases and the dissonance in Eq. (2) vanishes. Conversely, if the amplitudes of the partials increase, the dissonance increases. The point of maximum dissonance $d^{*}=0.24$ is derived directly from the model (1) above. The $s$ parameters in Eq. (3) allow a single functional form to interpolate between the various curves of Fig. 1 by sliding the dissonance curve along the frequency axis so that it begins at $f_{1}$, and by stretching (or compressing) it so that the maximum dissonance occurs at the appropriate frequency. A least-square fit was made to determine the values $s_{1}=0.021$ and $s_{2}=19$.

More generally, a (complex) timbre $F$ with base frequency $f_{1}$ is a collection of $n$ sine waves with frequencies $f_{1}<f_{2}<\cdots<f_{n}$ and amplitudes $v_{j}$ for $j=1,2, \ldots, n$. The partials will typically be displayed as the $n$-tuple $\left(f_{1}, f_{2}, \ldots, f_{n}\right)$. The dissonance of any timbre $F$ can be calculated as the sum of the dissonances of all pairs of partials

$$
D_{F}=\frac{1}{2} \sum_{i=1}^{n} \sum_{j=1}^{n} d\left(f_{i}, f_{j}, v_{i}, v_{j}\right) \text {. }
$$

Consider a fixed timbre $F$ with base frequency $f$. When two notes of this timbre are played simultaneously at an interval with ratio $\alpha$, the resulting sound has a dissonance that is the same as that of a timbre with frequencies $f_{i}$ and $\alpha f_{i}$ (with amplitudes $v_{j}$ ). Thus Eq. (5) can be used directly to calculate the dissonance between intervals (and chords) as well as the dissonance of isolated timbres. Defining the timbre $\alpha F$ to contain the frequencies $\left(\alpha f_{1}, \alpha f_{2}, \ldots, \alpha f_{n}\right)$ (with amplitudes $\left.v_{j}\right)$, the dissonance of the timbre $F$ at an interval $\alpha$ is

$$
D_{F}(\alpha)=D_{F}+D_{\alpha F}+\sum_{i=1}^{n} \sum_{j=1}^{n} d\left(f_{i}, \alpha f_{j}, v_{i}, v_{j}\right) .
$$

Finally, the dissonance curve generated by the timbre $F$ is defined as the function $D_{F}(\alpha)$ over all intervals of interest $\alpha$.

Figure 3 is the dissonance curve generated by the timbre of Fig. 2 over a range slightly larger than an octave, i.e., for $1 \leqslant \alpha \leqslant 2.2$. The model predicts that the most consonant interval is the unison, followed closely by the octave. Next is the fifth, followed by the fourth, the major third, the major sixth, and the minor third. These agree with standard musical usage. Looking at the data closely (see Table I) shows that the minima do not occur at exactly the scale steps of the 12-tone equal-tempered scale. Rather, they occur at the nearby simple ratios $1: 1,2: 1,3: 2$, $4: 3,5: 4$, and 5:3, respectively. These are exactly the locations of notes in the "justly intoned" scales. ${ }^{11}$ Thus qualitatively, dissonance calculations using harmonic timbres agree with musical experience and expectations.

It would be naive to suggest that truly musical properties can be measured in terms of a simple consonance. Even in the realm of harmony (and ignoring musically essential aspects such as melody and rhythm), consonance
TABLE I. Location of minima of Fig. $3, \beta={ }^{12} \sqrt{2}$.

\begin{tabular}{ccc}
\hline $\begin{array}{c}\text { Location of } \\
\text { minima }\end{array}$ & $\begin{array}{c}\text { Nearest 12-tone } \\
\text { scale step }\end{array}$ & $\begin{array}{c}\text { Nearest } \\
\text { ratio }\end{array}$ \\
\hline 1.20 & $\beta^{3}=1.189$ & $6: 5$ \\
1.25 & $\beta^{4}=1.159$ & $5: 4$ \\
1.33 & $\beta^{5}=1.335$ & $4: 3$ \\
1.40 & $\beta^{6}=1.414$ & $7: 5$ \\
1.50 & $\beta^{7}=1.498$ & $3: 2$ \\
1.67 & $\beta^{8}=1.587$ & $5: 3$ \\
1.75 & $\beta^{10}=1.782$ & $7: 4$ \\
2.00 & $\beta^{12}=2.000$ & $2: 1$ \\
\hline \hline
\end{tabular}

is not the whole story. Indeed, a harmonic progression that was uniformly consonant would likely be boring. Harmonic interest arises from a complex interplay of dissonance (restlessness) and consonance (rest).

Perhaps the most striking aspect of Fig. 3 is that many of the scale steps are coincident with local minima of the dissonance curve. Intervals which occur at points of local minima of the dissonance curve are perceived as relatively consonant. This observation forms the basis of the principle of local consonance. A timbre and a scale are said to be related if the timbre generates a dissonance curve whose local minima occur at scale positions.

Note that the shape of the dissonance curve is highly dependent on the frequencies (and amplitudes) of the components of the timbre. Changing these frequencies (and amplitudes) changes the location and depth of the local minima. By the principle of local consonance, this will change the optimum scale on which the timbre should be played. Thus we investigate scales and timbres which are related in the sense that local minima of the dissonance curve occur at (or very near) scale steps. The next section provides a detailed examination of the dissonance model.

\section{PROPERTIES OF DISSONANCE CURVES}

For certain simple timbres, dissonance curves can be completely characterized. This section derives bounds on the number and location of points of local consonance, and reveals some general properties. Two simplifications are made to streamline the discussion in this section. A single dissonance function is assumed for all frequencies, and all partials are presumed to have unit amplitudes. Thus $s=1$ in Eq. (3) and $v_{i j}=1$ in Eq. (4), simplifying Eq. (5) to Eq. (1) with $x=\left|f_{2}-f_{1}\right|$. Taking the derivative of Eq. (1) and setting it to zero shows that the point of maximum dissonance occurs when $d^{*}=[1 /(a-b)] \ln (a / b)$.

Theorem 1: For any timbre $F$ with partials at $\left(f_{1}, f_{2}, \ldots, f_{n}\right):$ (a) $\alpha^{*}=1$ is the global minimum of the dissonance curve $D_{F}(\alpha)$; (b) $\lim _{\alpha \rightarrow \infty} D_{F}(\alpha)=D_{F}+D_{\alpha F}$.

Proof: All proofs are relegated to the Appendix.

Part (b) shows that dissonance decreases as the interval $\alpha$ grows larger, approaching a value that is no more than the dissonance of the timbres $D_{F}$ and $D_{\alpha F}$ themselves. Part (a) affirms that the unison is the most consonant interval. Since $\alpha^{*}$ is the global minimum of $D_{F}(\alpha)$, all other minima must be local. The next several results seek to characterize these local minima. 
Theorem 2: Let timbre $F$ have partials at $\left(f_{1}, f_{2}\right)$ with $f_{2}-f_{1}>d^{*}$. Then the dissonance curve $D_{F}(\alpha)$ has a local minimum at $\alpha^{*}=f_{2} / f_{1}$.

This shows that the dissonance curve generated by a timbre with partials at $\left(f_{1}, f_{2}\right)$ has a point of local consonance when $\alpha^{*} f_{1}=f_{2}$. For example, for the timbre with partials at $(500,750), \alpha^{*}=1.5$. The result asserts that the timbre $\alpha^{*} F$, with frequencies $(750,1125)$ is a point of local consonance. In symbols, $D_{F}\left(\alpha^{*}-\epsilon\right)>D_{F}\left(\alpha^{*}\right)$ and $D_{F}\left(\alpha^{*}+\epsilon\right)>D_{F}\left(\alpha^{*}\right)$ for small $\epsilon$. Thus both $(748,1122)$ and $(752,1128)$ are less consonant than $(750,1125)$. This result is intuitively reasonable since when $\alpha f_{1} \neq f_{2}$, the dissonance between the partials at $\alpha f_{1}$ and $f_{2}$ is large, but when $\alpha f_{1}=f_{2}$, this term disappears from the consonance measure. Interestingly, the result fails when $f_{1}$ and $f_{2}$ are too close.

Theoren 3: Let timbre $F$ have partials $\left(f_{1}, f_{2}\right)$. Then there is a $\epsilon>0$ such that for $\left|f_{2}-f_{1}\right|<\epsilon$, the point $\alpha^{*}=f_{2} / f_{1}$ is not a local minimum of $D_{F}(\alpha)$.

In essence, if the partials $f_{1}$ and $f_{2}$ are too close, then the point of local consonance at $f_{2} / f_{1}$ disappears. Theorem 2 shows that a minimum occurs when partials coincide with each other. Minima can also occur when the partials are widely separated. For a two partial timbre $F$, suppose that $f_{2}-f_{1}>4 d^{*}$. Then there is a point of (local) maximum dissonance near $\alpha f_{1}=f_{1}+d^{*}$, and another near $\alpha f_{2}=f_{2}-d^{*}$. Consequently, there must be a minimum for some $\alpha$ between $\alpha_{L}=\left(f_{1}+d^{*}\right) / f_{1}$ and $\alpha_{H}=\left(f_{2}-d^{*}\right) / f_{2}$.

The full range of possible dissonance curves for two partial timbres is shown in Fig. 4. Figure 4(b) demonstrates the point of local consonance when $\alpha^{*}=f_{2} / f_{1}$ for moderate values of $f_{2}-f_{1}$. Figure 4(a) illustrates theorem 3 , for which $f_{2}-f_{1}$ is too small to allow a point of local consonance. Figure 4(c) shows the point of local consonance at $\alpha^{*}=f_{2} / f_{1}$, and also the minimum sandwiched between $\alpha_{L}$ and $\alpha_{H}$. Such minima are typically broad, and tend to disappear for timbres with more than a few partials. The dissonance curve of Fig. 3, for instance, consists exclusively of local minima caused by coinciding partials; the broad, in-between minima have vanished.

The next result describes points of local consonance for timbres with three partials.

Theorem 4: Let timbre $F$ have partials $\left(f_{1}, f_{2}, f_{3}\right)$. Then there are $c_{1}>0$ and $c_{2}>0$ such that whenever $f_{2}-f_{1}>d^{*}+c_{1}$ and $f_{3}-f_{2}>d^{*}+c_{2}$, local minima of the dissonance curve occur at $\alpha_{1}=f_{2} / f_{1}, \alpha_{2}=f_{3} / f_{1}$, and $\alpha_{3}=f_{3} / f_{2}$.

Thus timbres with three partials have three points of local consonance at $\alpha_{1} f_{1}=f_{2}, \alpha_{2} f_{1}=f_{3}$, and $\alpha_{3} f_{2}=f_{3}$, which are represented schematically in Fig. 5. Essentially, a minimum occurs whenever two of the partials coincide. Of course, other local minima may exist as well. Figure 6(a) shows the dissonance curve for the timbre $\left(f, \beta f, \beta^{4} f\right)$, where $\beta=\sqrt[10]{2}$. Note that the three minima predicted by theorem 4 are at exactly the first and fourth scale degrees of the ten-tone equal-tempered scale, and also at the "difference" frequency $\beta^{3} f$. Figure $6(\mathrm{~b})$ places the partials at $\left(f, \beta f, \beta^{6} f\right)$, generating the expected scale steps at 1 and 6 , and the difference $\beta^{5} f$ at step 5 . There is also a
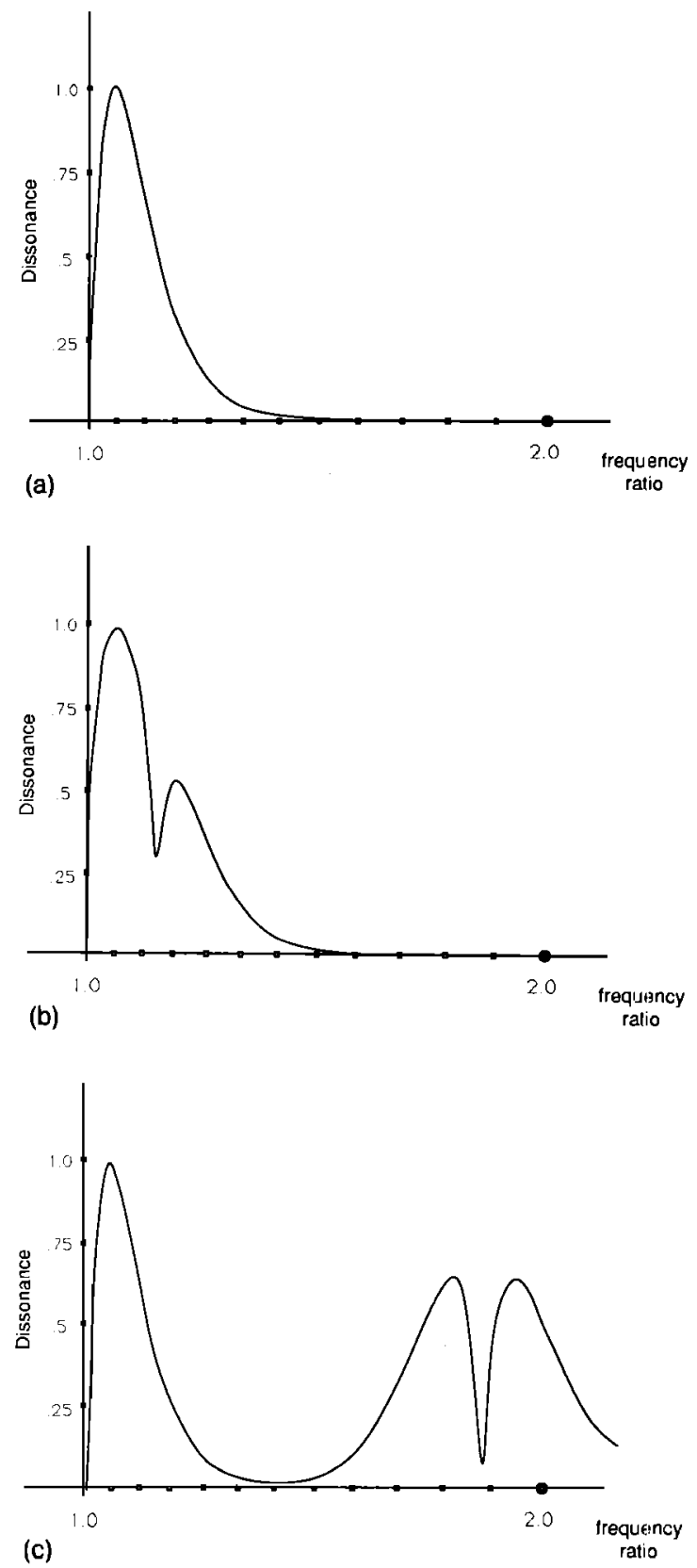

FIG. 4. Dissonance curves demonstrating the local minima of theorems 2 and 3. (a) For the timbre $(f, 1.01 f)$, the partials are too close to allow a point of local consonance. (b) Point of local consonance for timbre $(f, 1.15 f)$ occurs at $\alpha^{*}=1.15$. (c) Point of local consonance for timbre $(f, 1.86 f)$ at $\alpha^{*}=1.86$ and second "broad" minimum due to sparsity of partials.

"broad" equilibrium between the third and fourth steps, which is a result of the sparsity of the partials.

The final result of this section specifies the maximum number of points of local consonance of a timbre.

Theorem 5: Let timbre $F$ have partials $\left(f_{1}, f_{2}, \ldots, f_{n}\right)$. Then the dissonance curve generated by $F$ has at most $2 n(n-1)$ local minima.

The local minima are symmetrically located (on a logarithmic scale) so that half occur for $\alpha$ between 0 and 1 , and half occur for $\alpha$ between 1 and $\infty$. Half of the local minima are the broad type of Fig. $6(\mathrm{~b})$, while half occur when $f_{i}=\alpha f_{j}$ for some $i$ and $j$, which tend to be steep. Since the musically useful information is located for values 


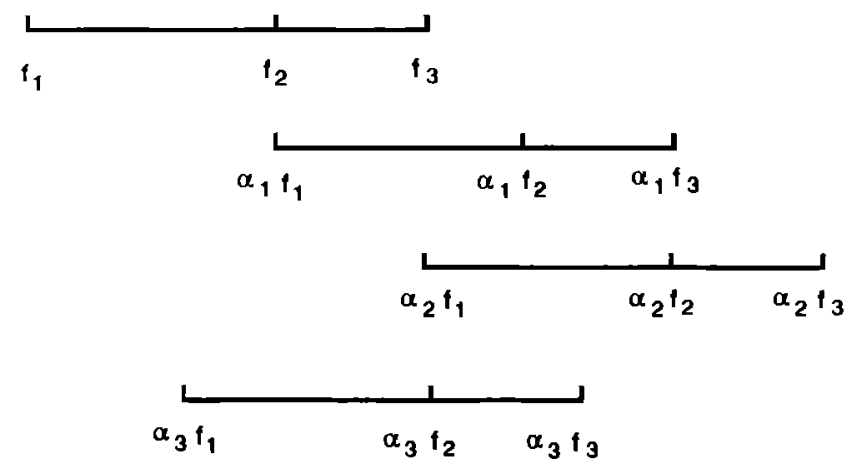

FIG. S. Schematic representation of three local minima of a three partial limbre.

of $\alpha$ near 1 or 2 , since the broad minima tend to vanish (except for sparse timbres), and since many minima are annihilated when partials are densely packed, (as in Fig. 3 ), typical dissonance curves exhibit far fewer than $2 n(n$ -1) local minima. In Fig. 3, for instance, there are only nine local minima within the octave of interest, considerably fewer than the theoretical maximum of $2 * 7 * 6=84$. It is possible to achieve the bound. For instance, the timbre $(f, 2 f, 3 f$ ) over the range $0<\alpha<6$ exhibits all 12 possible minima.

Despite the detail of this presentation, its main conclusion is not inaccessible: The most (musically) useful points of local consonance (i.e., local minima of the disso-
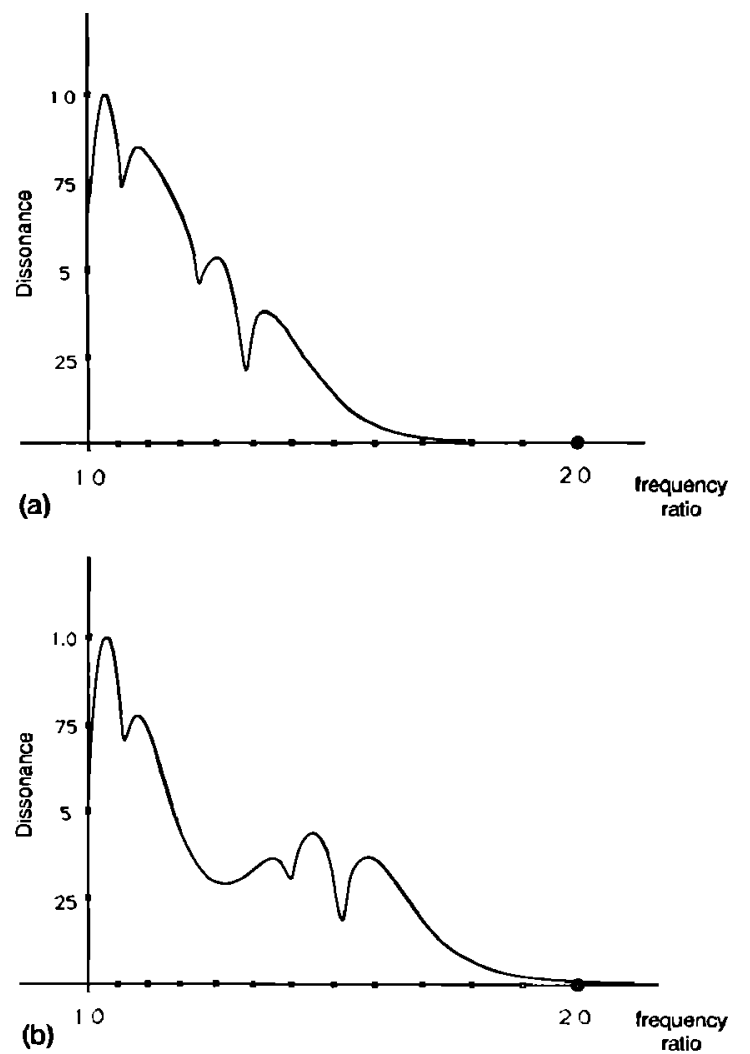

FIG. 6. Dissonance curves demonstrating the points of local consonance predicted by Theorem 4: (a) timbre $\left(f, \beta f, \beta^{4} f\right), \beta=\sqrt[10]{2}$. (b) timbre $\left(f, \beta f, \beta^{6} f\right)$. nance curve) tend to be located at intervals $\alpha$ for which $f_{i}=\alpha f_{j}$, where $f_{i}$ and $f_{j}$ are arbitrary partials of the timbre $F$.

The theorems of this section assumed that all partials were of equal amplitude. The effect of nonequal amplitudes is that some local minima may disappear, some may appear, and others may shift slightly in frequency. Fortunately, these changes occur in a structured way. To be concrete, let the timbre $F$ have partials $\left(f_{1}, f_{2}, \ldots, f_{n}\right)$ with amplitudes $\left(v_{1}, v_{2}, \ldots, v_{n}\right)$ and let $\hat{F}$ have the same set of partials but with amplitudes $(1,1, \ldots, 1)$. As discussed above, the dissonance curve for $\hat{F}$ will have up to $n(n-1)$ local minima due to coinciding partials which occur at the intervals $\alpha_{i j}=f_{i} / f_{j}$. As the amplitudes $v_{j}$ of $F$ move away from unity, the depth of the dissonance curve at $\alpha_{i j}$ may change and the local minima at some of the $\alpha_{i j}$ may disappear (an $\alpha_{i j}$ which is a local minimum of $\hat{F}$ may not be a local minimum of $F$ ) while other $\alpha_{i j}$ may appear (an $\alpha_{i j}$ which is not a local minimum of $\hat{F}$ may be a local minimum of $F$ ). Thus, amplitude variations of the partials tend to affect which of the $\alpha_{i j}$ happen to be local minima. The dissonance curve also contains up to $n(n-1)$ local minima of the "broad" type. The location of these equilibria are less certain, since they move continuously with respect to variations in the $v_{j}$.

\section{FROM TIMBRE TO SCALE}

This section constructs examples of scales appropriate for a variety of timbres, and explains various consonance related phenomena in terms of the principle of local consonance.

\section{A. Harmonic timbres}

Section I noted that the points of local consonance for the harmonic timbre with partials at $(f, 2 f, \ldots, 7 f)$ are located at simple integer ratios. The results of the previous section explain this elegantly. Candidate points of local consonance are at intervals $\alpha$ for which $f_{i}=\alpha f_{j}$. Since the partials are at integer multiples of $f, \alpha=n / m$ for integers $n$ and $m$ between 1 and 7. The principle of local consonance says that the most appropriate scale tones for harmonic timbres are located at such $\alpha$, and indeed, all the points of local consonance of Fig. 3 occur at such values, as tabulated in Table I. In a sense, this provides a physical basis for justly intoned scales.

\section{B. Stretched and compressed timbres}

Slaymaker ${ }^{6}$ and Mathews and Pierce ${ }^{7}$ have investigated timbres with partials at

$$
f_{j}=f A^{\log _{2} j} \text {. }
$$

When $A=2$, this is simply a harmonic timbre, since $f_{j}$ $=f 2^{\log _{2} j}=j f$. When $A<2$, the frequencies of the timbre are closer together than in harmonic timbres (compressed), while when $A>2$, the partials are further apart, or stretched. The most striking aspect of compressed and stretched timbres is the lack of a real octave. This can be seen clearly from the dissonance curves, which are plotted 


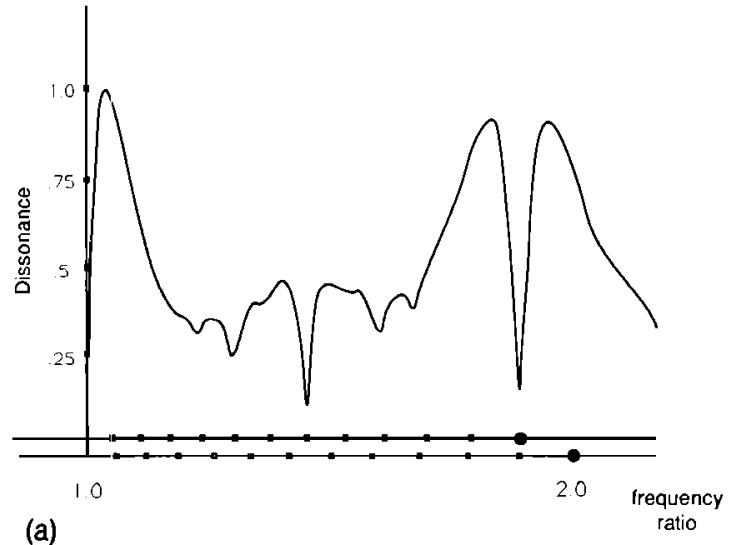

(a)

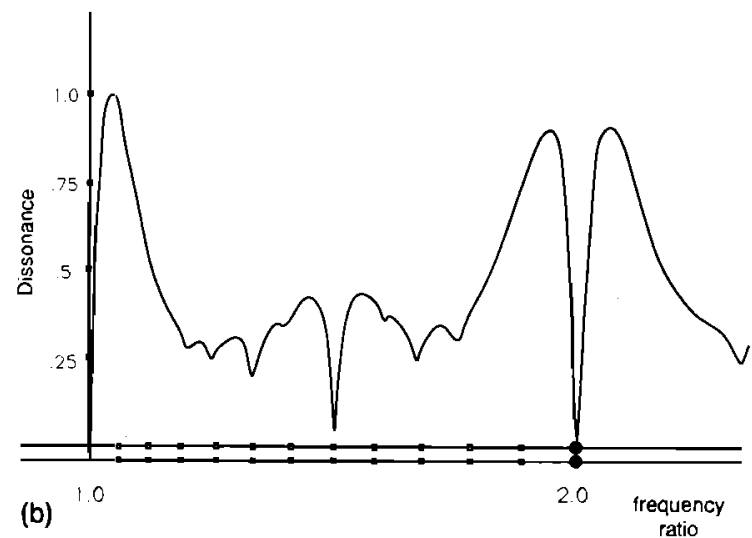

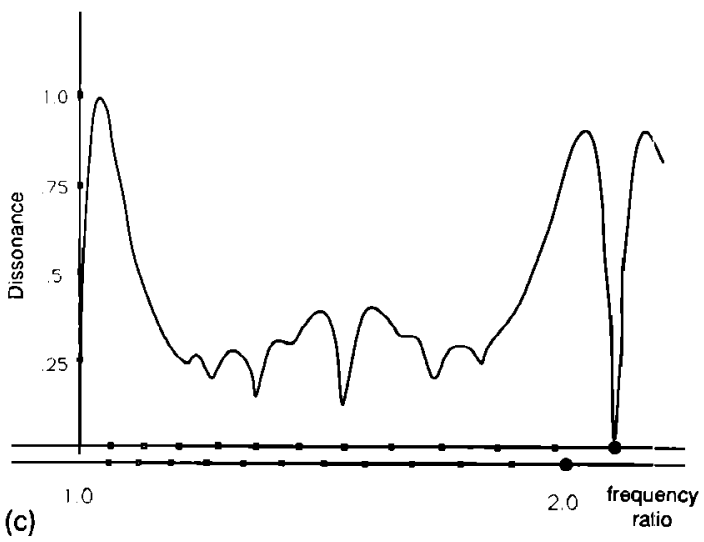

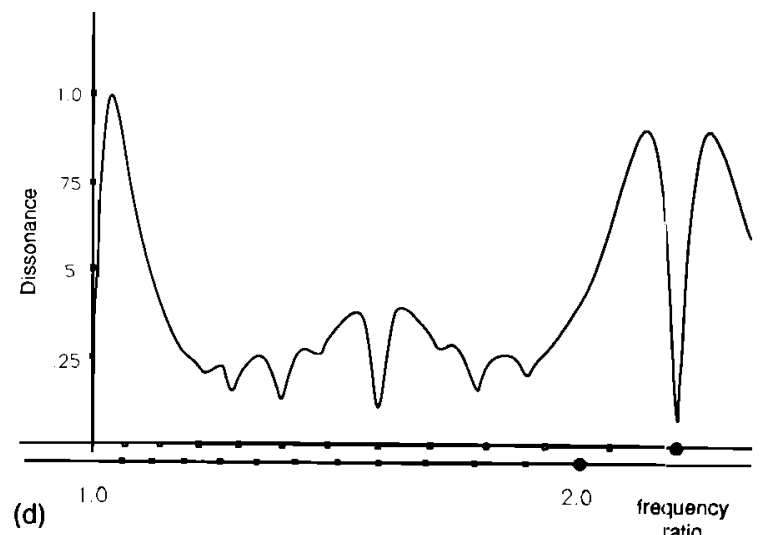

FIG. 7. Dissonance curves generated by stretched (and compressed) timbres. Each figure has two horizontal axes. The bottom axis shows the standard 12-tone equal-empered divisions of the octave (frequency ratio of 2:1). The top axis shows 12 equal divisions of the pseudo-octave with a frequency ratio of $A: 1$ : (a) $A=1.87$ (pseudo octave=major seventh), (b) $A=2$ (true octave), (c) $A=2.1$ (stretched octave), (d) $A=2.2$ (stretched further).

in Fig. 7(a)-(d) for $A=1.87,2.0,2.1$, and 2.2, respectively. In each case, the frequency ratio $A$ plays the role of the octave, which Mathews and Pierce call the pseudooctave. Real octaves sound dissonant and unresolved when $A \neq 2$ while the pseudo-octaves are highly consonant. More importantly, each curve has a similar contour. Points of local consonance occur at (or near) the twelve equal steps of the pseudo-octaves. "Pseudo-fifths," "pseudo-fourths," and "pseudo-thirds" are readily discernible. This suggests that much of music theory and practice can be transferred to compressed and stretched timbres, when played in compressed and stretched scales.

\section{A tuning for uniform beam instruments}

Percussion instruments containing uniform beams with free ends have partials that are not harmonically related. Indeed, considerable effort has been expended in an attempt to manipulate the shape of the beam to force it to vibrate harrnonically. ${ }^{12}$ The principle of local consonance suggests that there is a natural scale, defined by the timbre of the instrument, in which it will sound most consonant. The first seven frequencies of an ideal beam which is free to vibrate at both ends are given by Fletcher and Rossing ${ }^{13}$ as

$$
(f, 2.758 f, 5.406 f, 8.936 f, 13.35 f, 18.645 f, 24.82 f) \text {. }
$$

Two octaves of the dissonance curve for this timbre are shown in Fig. 8. The curve has numerous minima which are spaced unevenly at $1,1.27,1.33,1.4,1.49,1.65,1.96$, $2.09,2.25,2.47,2.76,3.05,3.24,3.45$, and 3.98 , which suggests that this would be the most natural sounding tuning for a such an instrument, at least in terms of consonance.

\section{Tuning for FM timbres}

One common method of sound synthesis is frequency modulation (FM) ${ }^{14}$ Noninteger ratios of the carrier and

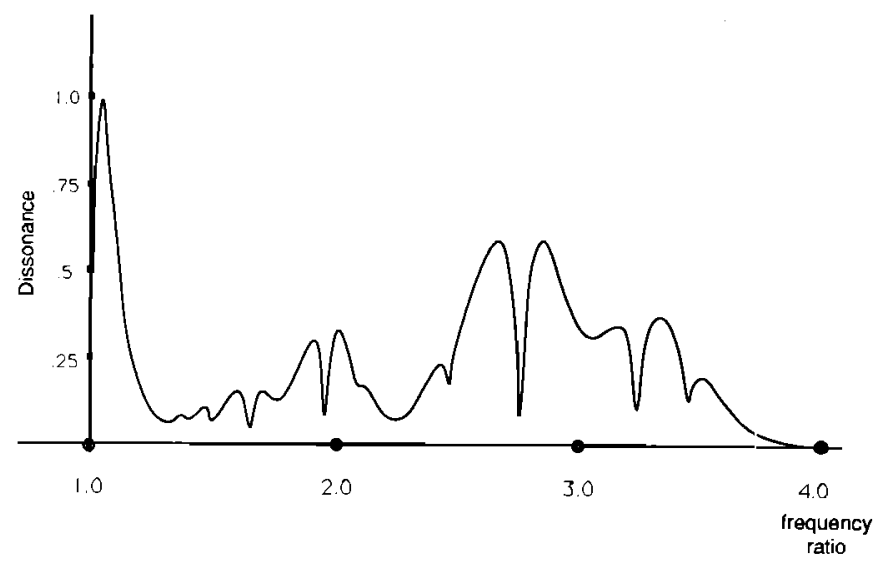

FIG. 8. Dissonance curve for uniform beam. 
TABLE II. Partials for the FM timbre with $c: m$ of 1:1.4 and modulating index $I=2$.

\begin{tabular}{lcc}
\hline \hline & Frequency & Amplitude \\
\hline$c-m$ & 0.4 & 0.57 \\
$c$ & 1.0 & 0.22 \\
$c-2 m$ & 1.8 & 0.35 \\
$c+m$ & 2.4 & 0.57 \\
$c-3 m$ & 3.2 & 0.13 \\
$c+2 m$ & 3.8 & 0.35 \\
$c-4 m$ & 4.6 & 0.03 \\
$c+3 m$ & 5.2 & 0.13 \\
$c+4 m$ & 6.6 & 0.03 \\
\hline \hline
\end{tabular}

modulating frequencies give nonharmonic timbres that are often relegated to percussive or bell patches because they sound dissonant when played in traditional 12-tone harmonies. The principle of local consonance suggests that such sounds can be played more harmoniously in scales which are determined by the timbres themselves.

For example, consider a simple FM tone with carrierto-modulation ratio $c: m$ of $1: 1.4$ and modulating index $I=2$. The frequencies and amplitudes of the resulting timbre are tabulated in Table II, and three octaves of the dissonance curve are plotted in Fig. 9. The appropriate scale notes for this timbre occur at the local minima of the dissonance curve, which can be read directly from the figure.

\section{FROM SCALE TO TIMBRE}

The optimal scale for a given timbre is found by locating the local minima of the dissonance curve. The complementary problem of finding an optimal timbre for a given scale, on the other hand, is poorly posed. This section demonstrates why there is no single "best" timbre for a given scale. "Locally best" timbres, however, can be specified as the solution to a certain multidimensional optimization problem which can be solved via global search methods such as the genetic algorithm. For certain types of scales (such as the $m$-tone equal-tempered scales) properties of the dissonance function can be exploited to bypass the search procedure.

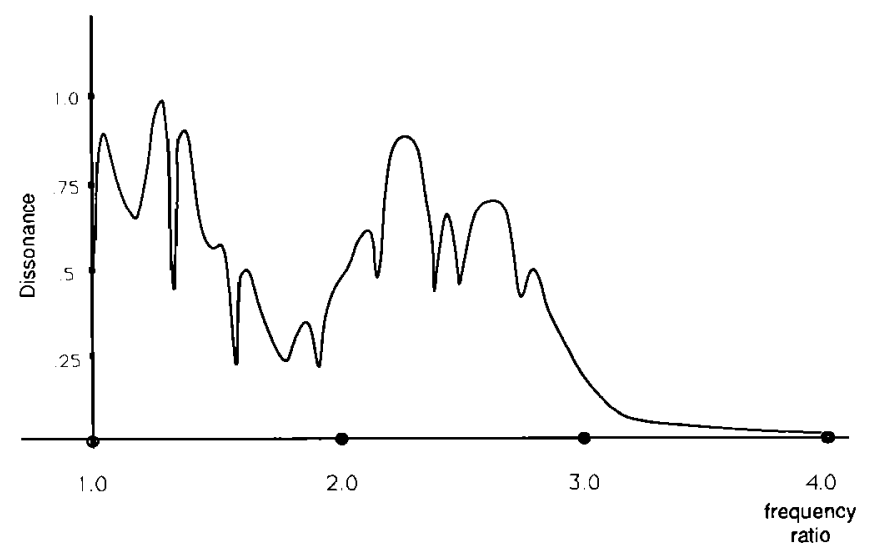

FIG. 9. Dissonance curve for FM timbre of Table II.

\section{A. Timbre selection as a constrained optimization problem}

Any set of $m$-scale tones specifies a set of $m-1$ intervals $\alpha_{1}, \alpha_{2}, \ldots, \alpha_{m-1}$. The problem of timbre selection is to choose a set of $n$ partials $\left(f_{1}, f_{2}, \ldots f_{n}\right)$ and amplitudes $\left(v_{1}, v_{2}, \ldots, v_{n}\right)$ to minimize the sum of the dissonances over the $m-1$ intervals. This criterion can be written

$$
J_{d}=\min _{\substack{\left(f_{1}, f_{2}, \ldots, f_{n}\right) \\\left(v_{1}, v_{2}, \ldots, v_{n}\right)}} \sum_{i=1}^{m-1} D_{F}\left(\alpha_{i}\right)
$$

where $D_{F}\left(\alpha_{i}\right)$ is the dissonance of the candidate timbre $F$ at the interval $\alpha_{i}$ as defined in Eq. (6). Recall that $D_{F}(\alpha)$ and hence $J_{d}$ are nonnegative for any $F$ and $\alpha$. Timbres which minimize Eq. (9) can approach zero dissonance in two ways: (1) Any timbre with $v_{1}=v_{2}=\cdots=v_{n}=0$ has $D_{F}\left(\alpha_{i}\right)=0$ for any $\alpha_{i}$. (2) Theorem 1 shows that for fixed and nonzero $v_{i}, D_{F}\left(\alpha_{i}\right)$ can be made arbitrarily small by choosing $\alpha_{i}$ large enough. Thus, if left unconstrained, the minimization of the criterion $J_{d}(9)$ will result in a trivial timbre via either (or both) of the above mechanisms. In order to avoid trivial solutions, some constraints are necessary.

A simple way to avoid (1) is to fix the $v_{i}$ a priori. Though not appealing, this is virtually necessary. For instance, suppose the $v_{i}$ for $i=1, \ldots, n-1$ were fixed while $v_{n}$ was allowed to vary. Then $J_{d}$ could always be reduced by choosing $v_{n}=0$. An alternative might be to fix the sum of the $v_{i}$, say $\Sigma v_{i}=v^{*}$. Again, $J_{d}$ could be reduced by setting $v_{j}=v^{*}$ and $v_{i}=0$ for all $i \neq j$.

One way to avoid the intervals $\alpha_{i}$ (and hence the frequencies of the partials) from escaping to infinity is to constrain them to some finite set. The cost $J_{d}$ will then be reduced by spreading the frequencies more or less evenly throughout the set, while trying to keep the cost low at $D_{F}\left(\alpha_{i}\right)$.

Fixing the $v_{i}$ and constraining the $\alpha_{i}$ to a finite set are enough to avoid trivial solutions, but they are not enough to provide good solutions. While the scale steps do tend to have reasonably small dissonance values, they tend not to occur at points of local consonance (i.e., at minima of the dissonance curves). The following criterion counts the number of $\alpha_{i}$ that are not minima:

$$
J_{m}=\min _{\left(f_{1}, f_{2}, \ldots, f_{n}\right)} \sum_{i=1}^{m-1} M_{F}\left(\alpha_{i}\right),
$$

where

$$
M_{F}\left(\alpha_{i}\right)= \begin{cases}0, & \text { if } D_{F}\left(\alpha_{i}+\epsilon\right)>D_{F}\left(\alpha_{i}\right) \\ & \text { and } D_{F}\left(\alpha_{i}-\epsilon\right)>D_{F}\left(\alpha_{i}\right), \\ 1, & \text { otherwise, }\end{cases}
$$

for some small $\epsilon>0$. Thus $M_{F}\left(\alpha_{i}\right)$ is 0 if $\alpha_{i}$ is a point of local consonance and is 1 otherwise. Clearly, $J_{m}$ alone would not be an appropriate criterion to minimize since it only reacts to the existence of minima and not to their actual value. The weighted sum of $J_{m}$ and $J_{d}$ 


$$
J=\min _{\left(f_{1}, f_{2}, \ldots, f_{n}\right)} \lambda_{1} \sum_{i=1}^{m-1} D_{F}\left(\alpha_{i}\right)+\lambda_{2} \sum_{i=1}^{m-1} M_{F}\left(\alpha_{i}\right)
$$

will tend to place the scale steps at local minima as well as to minimize the value of the dissonance curve. Through trial and error, we have found weightings of $\lambda_{2} / \lambda_{1} \approx 1000 / 1$ to be reasonable.

Minimizing $J$ of Eq. (12) is a $n$-dimensional optimization problem with a highly complex error surface. Gradient descent methods are unlikely to prove useful since they search only a small subset of the possible combinations of $f_{i}$. Random search methods such as simulated annealing or the genetic algorithm are slow, but they are global optimizers, at least asymptotically. We have found that the genetic algorithm (GA) often gives sensible answers. See Goldberg ${ }^{15}$ for a complete discussion of the algorithm.

Applications of the GA require that the problem be coded in a finite string called the "gene" and that a "fitness" function be defined. Genes for the timbre selection problem are formed by concatenating binary representations of the $f_{i}$, and the fitness function of the gene $\left(f_{1}, f_{2}, \ldots, f_{n}\right)$ is measured as the value $J$ of Eq. (12). Thus timbres are judged "more fit" if the cost $J$ is lower. The GA searches $n$-dimensional space measuring the fitness of timbres. The most fit are combined (via a "mating" procedure) into "child timbres" for the next generation. As generations pass, the algorithm tends to converge, and the most fit timbre is a good candidate for the minimizer of $J$. Indeed, the GA tends to return timbres which are well matched to the desired scale in the sense that scale steps tend to occur at points of local consonance and the total dissonance at scale steps is low. For example, when the 12-tone equal tempered scale is specified, the GA converges to harmonic timbres about $\frac{1}{5}$ of the time. This is a good indication that the algorithm is functioning and that the free parameters (the $\lambda$ 's) have been chosen sensibly.

\section{B. Timbres for an arbitrary scale}

As an example of the application of the genetic algorithm to the timbre selection problem, a desired scale was chosen with scale steps at $1,1.1875,1.3125,1.5,1.8125$, and 2. A set of amplitudes were chosen as $10,8.8,7.7,6.8$,

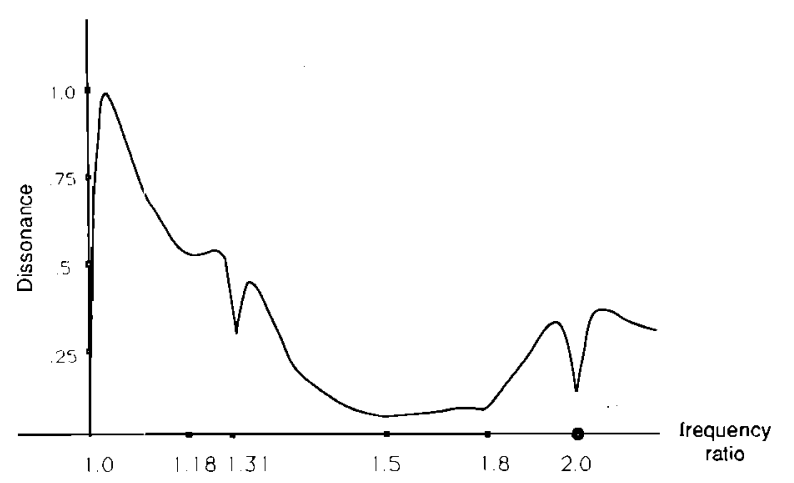

FIG. 10. Dissonance curve for timbre specified in Sec. IV B.
$5.9,5.2,4.6,4.0$, and the GA was allowed to search for the most fit timbre. The frequencies were coded as 8-bit binary numbers with 4 bits for the integer part and 4 bits for the fractional part. The best three timbres out of ten trial runs of the algorithm were

$(f, 1.8125 f, 4.9375 f, 14.0725 f, 9.875 f$,

$14.8125 f, 6.425 f, 12.96 f)$,

$(f, 1.5 f, 3.325 f, 10.255 f, 7.8125 f, 7.0975 f, 3.52 f, 3.875 f)$,

$(f, 2.39 f, 9.9275 f, 7.5625 f, 11.4025 f, 3.99 f$,

$6.37 f, 10.6025 f)$.

The dissonance curve of the best timbre is shown in Fig. 10. Clearly, this timbre is matched to the specified scale, since points of local consonance occur close to the scale steps.

\section{Timbres for equal temperaments}

For certain scales, such as the $m$-tone equal-tempered scales, properties of the dissonance curve can be exploited to quickly and easily design timbres, thus bypassing the need for an iterative solution to the constrained optimization problem (12).

Let $\beta=\sqrt[m]{2}$ be the ratio between successive scale steps, and consider timbres for which successive partials are ratios of $\beta^{i}$ for integers $i_{j}, j=1,2, \ldots, n$. Since $\beta^{m}=2$, there is an integer $k_{1}$ so that $\beta^{i_{j} / 2^{k_{1}}}=\beta^{k_{2}}$ for some $k_{2}$ between 0 and $m-1$. Thus, each partial, when transposed into the same octave as the fundamental, lies on a note of the scale. Such a timbre is said to be induced by the $m$-tone equaltempered scale. For example, harmonic timbres are induced timbres for the justly intoned scale.

Induced timbres are good candidate solutions to the optimization problem. Recall from Sec. II that points of local consonance tend to be located at intervals $\alpha$ for which $f_{i}=\alpha f_{j}$ where $f_{i}$ and $f_{j}$ are partials of the timbre $F$. Since the ratio between any pair of partials in an induced timbre is $\beta^{k}$ for some integer $k$, the dissonance curve will tend to have points of local consonance at such ratios; these ratios occur precisely at steps of the scale. Such timbres tend to minimize $J$ of Eq. (12) since the term $M_{F}\left(\beta^{k}\right)$ is zero whenever scale steps and points of local consonance coincide.

This insight can be exploited in two ways. First, it can be used to reduce the search space of the optimization routine. Instead of searching over all frequencies in a bounded region, the search need only be done over induced timbres. More straightforwardly, the timbre selection problem for equal-tempered scales can be solved by careful choice of induced timbres.

As an example, consider the problem of designing timbres to be played in ten-tone equal temperament. Ten-tone is often considered one of the worst temperaments for harmonic music, since the steps of the ten-tone scale are distinct from the (small) integer ratios, implying that harmonic timbres are very dissonant when played at intervals of $\beta^{k}$ for $\beta=\sqrt[10]{2}$. The principle of local consonance asserts 


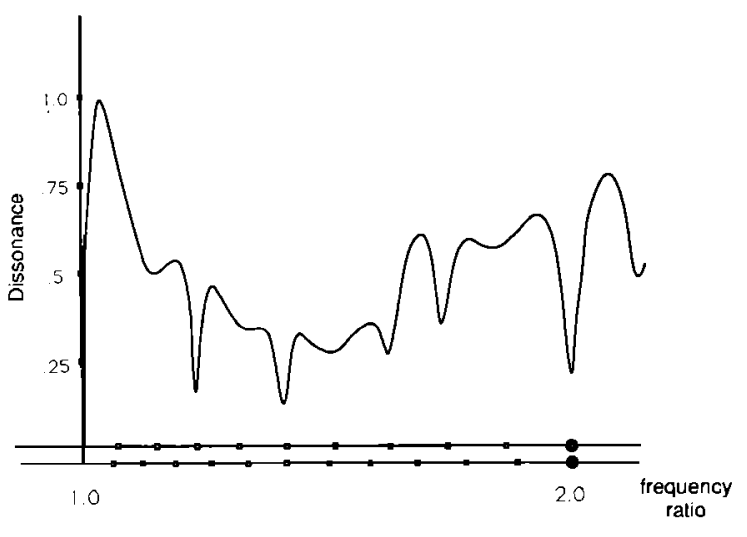

FIG. 11. Timbre designed to be played in ten-tone equal-tempered scale. Note that points of local consonance coincide with the ten-tone scale (top axis), but not with the 12-tone equal-tempered scale steps (bottom axis).

that these intervals will become consonant if played with correctly designed timbres. Here are three timbres induced by the ten-tone equal-tempered scale:

$$
\begin{aligned}
& \left(f, \beta^{10} f, \beta^{17} f, \beta^{20} f, \beta^{25} f, \beta^{28} f, \beta^{30} f\right), \\
& \left(f, \beta^{7} f, \beta^{16} f, \beta^{21} f, \beta^{24} f, \beta^{28} f, \beta^{37} f\right), \\
& \left(f, \beta^{7} f, \beta^{13} f, \beta^{17} f, \beta^{23} f, \beta^{28} f, \beta^{30} f\right) .
\end{aligned}
$$

The dissonance curve of the first timbre is shown in Fig. 11.

\section{DISCUSSIONS AND CONCLUSIONS}

This paper developed the principle of local consonance and proposed two complementary computational techniques: A way to find consonant scales given a specified timbre, and a way to find consonant timbres given a specified scale. Local consonance is based on an explicit parametrization of Plomp and Levelt's consonance curves. By exploiting this parametrization, several concrete results were derived, which describe in generic terms the number and location of local minima of the consonance curves. These results explain certain consonance related phenomena and simplify the timbre selection problem. One implication is that the musical notion of consonance of intervals such as the octave and fifth can be viewed as a result of the timbre of the tones we listen to. The justly intoned scales can similarly be viewed as a consequence of the harmonic timbres of musical instruments.

The consonance theory of Plomp and Levelt is probably the most important current consonance theory, but it is not uncontroversial. In the long run, the importance and usefulness of the timbre-scale relationship presented here depends on the validity and power of the Plomp-Levelt theory. Conversely, the computational techniques of this paper can be viewed as a way to apply the Plomp-Levelt results to nonharmonic music and instrument design. $\mathrm{Mu}-$ sic composed using related timbres and scales provides indirect support for the Plomp-Levelt consonance theory.

The results of Sec. II show some of the generic properties of dissonance curves. The first theorem says that the unison is always the most consonant interval for any tim- bre, and that dissonance always decreases as the interval increases, at least for very large intervals. A tuba and a piccolo may play together without fear of dissonance. Theorems 2 and 4 show that local minima often occur at intervals which are ratios of the frequencies of the partials. The local minima corresponding to such coinciding partials are good candidates for intervals of a scale while the broad minima due to wide separation of partials tend to vanish, at least for complex timbres. The final theorem gives a bound on the number of points of local consonance in terms of the complexity of the timbre.

Theorem 3 suggests that local minima of the dissonance curve are unlikely for intervals larger than about half the interval $d^{*}$ at which maximum dissonance occurs. Plomp and Levelt estimate that $d^{*}$ corresponds to a little less than $\frac{1}{3}$ of the critical bandwidth. Thus theorem 3 predicts that scale steps closer together than about $\frac{1}{6}$ of the critical bandwidth should be rare. Plomp and Levelt show that adjacent partials in a Bach sonata fall between critical bandwidth and about $\frac{1}{4}$ critical bandwidth. It would be interesting to conduct studies on the average distance between partials in nonwestern music.

The principle of local consonance suffers from the limitations of the model used to calculate the dissonance curves. It is only valid for "reasonable" frequencies and amplitudes. Frequencies outside the range of audibility, for instance, cannot contribute to dissonance. Even within the audible range, there is considerable variation in the frequency response of the ear. To compensate, the amplitudes of the partials could be weighted by the inverse of the Fletcher-Munson curves, ${ }^{16}$ but this has not been pursued here in order to retain the (relative) simplicity of the model. Similarly, the amplitudes must not be loud enough to introduce significant nonlinear distortions in the ear. ${ }^{17}$

The problem of timbre selection for a specified set of scale tones is posed as a multidimensional optimization problem, and can be solved by "random search" methods. Because the optimization must be constrained to avoid trivial solutions, the amplitudes are specified a priori, limiting the selection of timbres to a choice of frequencies. Different timbres with different mixes of frequencies and amplitudes may solve the optimization problem equally well, implying that they are equivalent in terms of consonance.

The principle of local consonance makes concrete predictions about the consonance of related timbres and scales, and these must be verified by controlled listener tests. Experiments which would validate (or falsify) the principle might involve fixing some nonharmonic timbre, and finding the most consonant intervals (or scales) from the dissonance curve. Listeners could then judge the relative consonance of these intervals (or scales) when interspersed among a number of other intervals (or scales). Another type of test would be to create a large number of nonharmonic timbres. Listeners could then be asked to order the timbres in terms of consonance, and the ordering compared to the predictions of the theory.

All of the timbres mentioned in this paper were generated on a personal computer and downloaded to an En- 
soniq EPS sampler, where the appropriate scale could be easily defined in a "tuning table." Informal listening tests (using colleagues and friends) upheld the essential predictions of the principle of local consonance. One of the most convincing tests involved the stretched timbres of Sec. III B. Two tones were generated which were identical in all parameters except that one used $A=2.0$ (a normal harmonic timbre) and the other was stretched to $A=2.1$ [as in Fig. 7(c)]. Trwo scales were used, a standard 12-tone equaltempered scale, and a scale stretched so that the pseudooctave occurred at 2.1. The four possibilities were compared: (1) $A=2.0$ timbre with standard scale, (2) $A=2.1$ timbre with standard scale, (3) $A=2.0$ timbre with stretched scale, (4) $A=2.1$ timbre with stretched scale.

A list of adjectives was placed before the listeners (who were not told the purpose of the test) and they were asked to check those that applied. Overwhelmingly, cases (1) and (4) were described as pleasing, consonant, and musical, while (2) and (3) were described as dissonant, ugly, unmusical, and out-of-tune. Some of the more sophisticated listeners (i.e., those with significant musical training) also described (4) as out-of-tune. A similar test was conducted with the timbres of Sec. IV C which were specially designed for ten-tone equal temperament. Again, the related scales and timbres were judged consonant while the unrelated scales and timbres (such as the ten-tone timbre played in the 12-tone scale, and the 12-tone timbre played in the ten-tone scale) were judged dissonant.

Such informal tests cannot be taken too seriously, but they do indicate the need for more rigorous experiments. One subtlety that arises when dealing with nonharmonic partials is that it is important for the partials to fuse, to be perceived holistically as a single entity rather than as a collection of oddly placed sine waves. Fusing is greatly helped by commonalities in the partials: Similar pitch vibrato, similar amplitude fluctuations, similar onset time, and similar envelopes. Whatever the experiment, care must be taken that the timbres fuse properly.

Anotherr way to test the predictions of the principle of local consonance is to investigate the relationship between timbre and scale in other musical cultures. For instance, the gamelon orchestras of Indonesia contain ensembles of metalophone instruments of various sizes, materials, and timbres. There are as many different gamelon tunings as there are gamelon orchestras because instruments in the Indonesian musical tradition are not tuned to a single standard reference. Rather, each instrument is tuned and timbrally adjusted to work in a particular orchestral context. ${ }^{11}$ It would be an important test for the theory to measure the spectra of the instruments of various gamelons and to compare the scale predicted by the dissonance curves to the actual scales used.

The advent of inexpensive musical synthesizers capable of realizing arbitrary sounds allows exploration of nonharmonic acoustic spaces, and the principle of local consonance provides guidelines on how to sensibly relate scale and timbre. More ambitiously, it is easy to imagine a whole orchestra of nonharmonic instruments capable of playing consonant music. The computational techniques of this pa- per allow specification of timbres and scales for such instruments. An important area for further investigation is how to engineer acoustic instruments so as to play in specified (nonharmonic) timbres and in related tunings. Ultimately, the test of the theory is whether it will be used to make music.

\section{ACKNOWLEDGMENTS}

The author would like to thank Tom Staley for extensive discussions on tunings, tonality, and timbre. D. Hall, B. Hopkin, W. Strong, and two anonymous reviewers were helpful in commenting on various versions of this paper.

\section{APPENDIX}

Proofs of the theorems follow. Throughout this Appendix, the dissonance curve (6) is written

$$
D_{F}(\alpha)=D_{F}+D_{\alpha F}+\sum_{i=1}^{n} \sum_{j=1}^{n} d\left(f_{i}, \alpha f_{j}\right),
$$

where the last two (amplitude) arguments of Eq. (2) have been abandoned since $v_{i}=1$ is assumed for all $i$ and $s=1$ is also assumed. When needed, the values $a=3.5$ and $b=5.75$ are used, though generally $b>a>0$ is enough.

Theorem 1: For any timbre $F$ with partials at $\left(f_{1}, f_{2}, \ldots, f_{n}\right)$ : (a) $\alpha^{*}=1$ is the global minimum of the dissonance curve $D_{F}(\alpha)$; (b) $\lim _{\alpha \rightarrow \infty} D_{F}(\alpha)=D_{F}+D_{\alpha F}$.

Proof of (a): For all $\alpha, D_{F}=D_{\alpha F}$. The function (1) has its global minimum at $x=0$ since $b>a>0$. Thus $\sum_{i=1}^{n} \sum_{j=1}^{n} d\left(f_{i}, \alpha f_{j}\right)>2 D_{F} \quad$ whenever $\quad \alpha \neq 1, \quad$ and $\sum_{i=1}^{n} \Sigma_{j=1}^{n} d\left(f_{i}, \alpha f_{j}\right)=2 D_{F}$ for $\alpha=1$. Consequently, $D_{F}(1)=4 D_{F}<D_{F}(\alpha)$ for all $\alpha \neq 1$.

Proof of (b): Clearly, $d(x) \rightarrow 0$ as $x \rightarrow \infty$. Thus $d\left(f_{i}, \alpha f_{j}\right) \rightarrow 0$ for all $i, j$ as $\alpha \rightarrow \infty$. Consequently, $D_{F}(\alpha) \rightarrow D_{F}+D_{\alpha F}$ as $\alpha \rightarrow \infty$.

Theorem 2: Let timbre $F$ have partials at $\left(f_{1}, f_{2}\right)$ with $f_{2}-f_{1}>d^{*}$. Then the dissonance curve $D_{F}(\alpha)$ has a local minimum at $\alpha^{*}=f_{2} / f_{1}$.

Proof: Let timbre $G$ have partials $\left(g_{1}, g_{2}\right)=\left(\alpha f_{1}, \alpha f_{2}\right)$. Then $D_{F}=D_{G}=D_{\alpha F}$, and any change in $D_{F}(\alpha)$ must arise from the double sum in Eq. (A1), which contains the terms $d\left(f_{i}, g_{j}\right)$ for $i=1,2$ and $j=1,2$. For $\alpha^{*}==f_{2} / f_{1}$, $\left(g_{1}, g_{2}\right)=\left(f_{2}, \alpha f_{2}\right)$. As $\alpha$ is perturbed from $\alpha^{*}$, the contribution from the term $d\left(f_{2}, g_{1}\right)=d\left(f_{2}, \alpha f_{1}\right)$ increases, since at $\alpha^{*}, \alpha^{*} f_{1}=f_{2}$ and so $d\left(f_{2}, g_{1}\right)=d\left(f_{2}, f_{2}\right)=0$. Thus the result can be demonstrated by showing that the increase in $d\left(f_{2}, g_{1}\right)$ is greater than the decrease in the other three terms combined. The increase in $d\left(f_{2}, g_{1}\right)$ is proportional to

$$
d^{\prime}(0)=-a e^{-a x}+\left.b e^{-b x}\right|_{x=0}=b-a .
$$

Since $f_{2}-f_{1}>d^{*}$, the decrease in $d\left(f_{1}, g_{1}\right)$ can be bounded by the slope at the point $x^{* *}$ on $d(x)$ where $d^{\prime}\left(x^{* *}\right)$ is negative and of largest absolute value. This occurs when $d^{\prime \prime}\left(x^{* *}\right)=0$. Since

$$
d^{\prime \prime}(x)=a^{2} e^{-a x}-b^{2} e^{-b x},
$$

$x^{* *}=2 /(a-b) \ln (a / b)$. After some simplification, 


$$
d^{\prime}\left(x^{* *}\right)=(a / b)^{(b+a) /(b-a)}(a-b)=k(a-b) .
$$

Thus the slope at $x^{* *}$ is $k$ times the slope at 0 . For $a=3$, $b=6, k=\frac{1}{8}$. Hence the increase in $d\left(f_{2}, g_{1}\right)$ is eight times larger than the decrease in $d\left(f_{1}, g_{1}\right)$. But $\left|d\left(f_{1}, g_{1}\right)\right|$ is approximately equal to $\left|d\left(f_{2}, g_{2}\right)\right|$, and is greater than $\left|d\left(f_{1}, g_{2}\right)\right|$. This proves the desired result.

Theorem 3: Let timbre $F$ have partials $\left(f_{1}, f_{2}\right)$. Then there is a $\epsilon>0$ such that for $\left|f_{2}-f_{1}\right|<\epsilon$, the point $\alpha^{*}=f_{2} / f_{1}$ is not a local minimum of $D_{F}(\alpha)$.

Proof: Define $G$ as in theorem 2. Again, any change in $D_{F}(\alpha)$ is a result of the four terms in the sum of (A1). For small $\epsilon>0$, note that: $d\left(f_{1}, g_{1}+\epsilon\right)>d\left(f_{1}, g_{1}\right)$ $>d\left(f_{1}, g_{1}-\epsilon\right), d\left(f_{1}, g_{2}+\epsilon\right)>d\left(f_{1}, g_{2}\right)>d\left(f_{1}, g_{2}-\epsilon\right)$, $d\left(f_{2}, g_{2}+\epsilon\right)>d\left(f_{2}, g_{2}\right)>d\left(f_{2}, g_{2}-\epsilon\right)$, and $d\left(f_{2}, g_{1}+\epsilon\right)$ $>d\left(f_{2}, g_{1}\right)$.

On the other hand, $d\left(f_{2}, g_{1}-\epsilon\right)>d\left(f_{2}, g_{1}\right)$ $=d\left(f_{2}, f_{2}\right)=0$. For small $\epsilon$, the change in all four terms is approximately $\epsilon(b-a)$ in magnitude. Thus the dissonance value is decreased as $G$ is moved $\epsilon$ closer to $F$, and $\alpha^{*}=f_{2} / f_{1}$ is not a minimum.

Theorem 4: Let timbre $F$ have partials $\left(f_{1}, f_{2}, f_{3}\right)$. Then there are $c_{1}>0$ and $c_{2}>0$ such that whenever $f_{2}-f_{1}>d^{*}+c_{1}$ and $f_{3}-f_{2}>d^{*}+c_{2}$, local minima of the dissonance curve occur at $\alpha_{1}=f_{2} / f_{1}, \quad \alpha_{2}=f_{3} / f_{1}$, $\alpha_{3}=f_{3} / f_{2}$.

Proof: Let $G$ have partials $\left(g_{1}, g_{2}, g_{3}\right)$ $=\left(\alpha f_{1}, \alpha f_{2}, \alpha f_{3}\right)$. Suppose first that $f_{2}-f_{1}>f_{3}-f_{2}+c_{2}$. Consider the candidate minimum $\alpha_{1}$. For small $\epsilon$, the most significant terms in $D_{F}(\alpha+\epsilon)-D_{F}(\alpha)$ are $d\left(f_{2}, g_{1}\right)$ and $d\left(f_{3}, g_{2}\right)$, since all others are separated by at least $d^{*}+c_{2}$. For $\quad \epsilon>0, \quad d\left(f_{2}, g_{1}+\epsilon\right)>d\left(f_{2}, g_{1}\right), \quad d\left(f_{3}, g_{2}+\epsilon\right)$ $>d\left(f_{3}, g_{2}\right)$, and $d\left(f_{2}, g_{1}-\epsilon\right)>d\left(f_{2}, g_{1}\right)$. On the other hand, $d\left(f_{3}, g_{2}-\epsilon\right)<d\left(f_{3}, g_{2}\right)$. But $d^{\prime}(0)=b-a$ and $d^{\prime \prime}(0)=a^{2}-b^{2}<0$, so the slope is decreasing. Hence $\left|d\left(f_{2}, g_{1}-\epsilon\right)\right|>\left|d\left(f_{3}, g_{2}-\epsilon\right)\right|$. Consequently, $D_{F}\left(\alpha_{1}+\epsilon\right)>D_{F}\left(\alpha_{1}\right)$ and $D_{F}\left(\alpha_{1}-\epsilon\right)>D_{F}\left(\alpha_{1}\right)$, showing that $\alpha_{1}$ is a local minimum. The case $f_{3}-f_{2}>f_{2}-f_{1}+c_{1}$ follows identically. The proofs for $\alpha_{2}$ and $\alpha_{3}$ are similar. $\square$

Theorem 5: Let timbre $F$ have partials $\left(f_{1}, f_{2}, \ldots, f_{n}\right)$. Then the dissonance curve generated by $F$ has at most $2 n(n-1)$ local minima.
Proof: Consider the portion of $D_{F}(\alpha)$ due to the partial $f$ interacting with a fixed partial $f_{j}$. For small $\alpha$, $\alpha f \ll f_{j}$ and $d\left(\alpha f, f_{j}\right) \approx 0$. As $\alpha$ increases, $d\left(\alpha f, f_{j}\right)$ increases until $\alpha f=f_{j}$, where $d\left(\alpha f, f_{j}\right)=0$. As $\alpha$ increases further, $d\left(\alpha f, f_{j}\right)$ increases to a maximum at $\alpha f=d^{*}$. Finally, as $\alpha \rightarrow \infty, d\left(\alpha f, f_{j}\right) \rightarrow 0$. Thus $f$ interacting with a fixed $f_{j}$ has two maxima and one minima. Each $f_{i}$ can interact with each $f_{j}$, and there are $n^{2}-n$ distinct pairs. Since $D_{F}(\alpha)$ consists of $n(n-1)$ such curves added together, there are at most $2 n(n-1)$ maxima. Consequently, there are at most $2 n(n-1)$ minima.

'W. Carlos, "Tuning: At the Crossroads," Comput. Music J. 11, 29-43 (Spring, 1987).

${ }^{2}$ R. Plomp and W. J. M. Levelt, "Tonal Consonance and Critical Bandwidth," J. Acoust. Soc. Am. 38, 548-560 (1965).

${ }^{3}$ H. Helmholtz, On the Sensations of Tones (Dover, New York, 1954).

${ }^{4}$ A. Kameoka and M. Kuriyagawa, "Consonance Theory Part II: Consonance of complex Tones and its calculation method," J. Acoust. Soc. Am. 45, 1460-1469 (1968).

${ }^{5} J$. R. Pierce, "Attaining Consonance in Arbitrary Scales," J. Acoust. Soc. Am. 40, 249 (1966).

${ }^{6}$ F. H. Slaymaker, "Chords from tones having stretched partials," J. Acoust. Soc. Am. 47, 1469-1571 (1968).

${ }^{7}$ M. V. Mathews and J. R. Pierce, "Harmony and Nonharmonic Partials," J. Acoust. Soc. Am. 68, 1252-1257 (1980).

${ }^{8} \mathrm{~J}$. M. Geary, "Consonance and Dissonance of Pairs of Inharmonic Sounds," J. Acoust. Soc. Am. 67, 1785-1789 (1980).

${ }^{9}$ M. V. Mathews, J. R. Pierce, A. Reeves, and L. A. Roberts, "Theoretical and experimental explorations of the Bohlen-Pierce scale," J. Acoust. Soc. Am. 84, 1214-1222 (1988).

${ }^{10}$ W. A. Sethares, "Relating Timbre and Scale," Exp. Musical Instrum. 9(1) (Sept. 1993).

${ }^{11}$ S. R. Wilkinson, Tuning In (Hal Leonard, Milwaukee, WI, 1988).

${ }^{12} \mathrm{~F}$. Orduna-Bustamante, "Nonuniform beams with harmonically related overtones for use in percussion instruments," J. Acoust. Soc. Am. 90, 2935-2941 (1991).

${ }^{13}$ N. H. Fletcher and T. D. Rossing, The Physics of Musical Instruments (Springer-Verlag, Berlin, 1991).

${ }^{14} \mathrm{~J}$. M. Chowning, "The Synthesis of Complex Audio Spectra by Means of Frequency Modulation," J. Audio Eng. Soc. 21, 526-534 (1973).

${ }^{15} \mathrm{~S}$. Goldberg, Genetic Algorithms in Search, Optimization, and Machine Learning (Addison-Wesley, New York, 1989).

${ }^{16} \mathrm{H}$. Fletcher and W. A. Munson, "Loudness, its definition, measurement, and calculation," J. Acoust. Soc. Am. 5, 82 (1933).

${ }^{17}$ D. M. Green, An Introduction to Hearing (Wiley, New York, 1976). 